\title{
Faces of Privacy: Effect of Culture and Context
}

\author{
Kari-Jouko Räihä and Saila Ovaska \\ Unit for Computer Human Interaction (TAUCHI), Department of Computer Sciences \\ FIN-33014 University of Tampere, Finland \\ \{kari-jouko.raiha, saila.ovaska\}@cs.uta.fi
}

\begin{abstract}
We repeated in Finland a study on privacy concerns originally carried out in the USA. The results suggest that there are cultural differences in the willingness of people to reveal privacy-sensitive information.
\end{abstract}

Keywords: Privacy, regulation mechanisms, cultural differences.

\section{Introduction}

It has been suggested [1] that mobile context-aware telephones could be designed to show the location information of the callee, who could set the phone to automatically dismiss the call still providing some information about his or her current situation. However, for different callers there would be a need to show different level of detail. For instance, a call from the boss requires more precise detail than a call from a merchant, especially if it comes during working hours. Also the presence of other people is an important message to the caller. Lederer and his colleagues combined these aspects into a metaphorical "Face" shown to the caller [1].

We repeated the data collection of [2] in Finland. We point at preliminary findings about possible cultural variation: based on the data collected, we expect that privacy regulation mechanisms may need to be culturally varied.

\section{The Study}

Data collection in the original study [2] was done with a web questionnaire. The questionnaire introduced a background scenario where the respondent was asked to imagine having a mobile phone with identity and profile information that could be disclosed to the callers with various levels of accuracy:

- Three faces: True, Vague, Blank, each revealing a different level of detail. For instance, a True face would reveal the actual identity of the person, a Vague face would show a pseudonym, and a Blank face would be anonymous. Similar distinctions applied to profile (contact information and interests), activity, and location.

Furthermore, the subjects were asked to fill in the face matching their preferences in $2 \times 4=8$ different contexts.

- Two situations: Working Lunch, Social Evening

- Four inquirers: Spouse/significant other, Employer, Stranger, Merchant. 
The original study was part of a more extensive user-centered design process [3]. The web survey was preceded by interviews and followed by prototype evaluations to guide the design of the privacy regulation mechanism. In addition to serving this ultimate purpose, the specific question answered through the web survey was: Which is more important, the inquirer or the situation in which the information is disclosed?

This question was introduced to students in an advanced HCI class in Finland in 2004 and 2005. They were asked to interview 3-5 friends or acquaintances and report by email the faces chosen by the respondents and possible comments they had. The students of the class came from different ethnic backgrounds. Based on a preliminary analysis of the data collected in 2004 we asked the course in 2005 to report also the cultural background of the persons they interviewed. The demographic variables collected were gender, nationality, occupation, and age group. Students were used for data collection to teach them about privacy issues and to increase the sample size.

\section{The Results and Analysis}

The fundamental design-oriented question of interest in the original study [2] was whether people would use the faces at all, i.e., how many faces would they use overall. The resulting mean number of faces presented across situations and inquirers is shown in Table 1, both for the original study and our students. From the three possible faces, the respondents selected on average more than two different faces to display in the described events.

Table 1. Mean number of different faces used overall in the eight contexts

\begin{tabular}{lcc}
\hline & Original study $(\mathrm{n}=130)$ & Our study $(\mathrm{n}=246)$ \\
\hline Working lunch & $2.72(\operatorname{sd} 0.84)$ & $2.37(\operatorname{sd} 0.59)$ \\
Social evening & $2.58(\operatorname{sd} 0.89)$ & $2.35(\operatorname{sd~} 0.59)$ \\
\hline
\end{tabular}

The main observation from these results is that within a given situation, subjects did vary faces across inquirers. Our numbers are lower than the original ones, but still indicate the interest in using a variety of faces.

Lederer et al. [2] found that for a given inquirer, subjects generally did not vary faces across situations. Their conclusion was that the inquirer's identity is a stronger determinant of privacy preferences than the user's situation. Table 2 summarizes the data when responses for both situations were pulled together.

Table 2. Face variation based on situation

\begin{tabular}{l|rc|cc}
\hline & \multicolumn{2}{|c|}{ Original study } & \multicolumn{2}{c}{ Our study in 2004 } \\
\hline Inquirer & Same Face & Different Face & Same Face & Different Face \\
\hline Spouse & $83.8 \%$ & $16.2 \%$ & $84.6 \%$ & $15.4 \%$ \\
Employer & $54.6 \%$ & $45.4 \%$ & $37.8 \%$ & $66.2 \%$ \\
Stranger & $77.7 \%$ & $22.3 \%$ & $80.9 \%$ & $19.1 \%$ \\
Merchant & $86.2 \%$ & $13.6 \%$ & $79.3 \%$ & $20.7 \%$ \\
\hline
\end{tabular}


The results are similar, but there are marked differences as well, particularly in the case where the inquirer is the employer or merchant. Figure 1 shows a more detailed distribution of the different faces for these two cases.

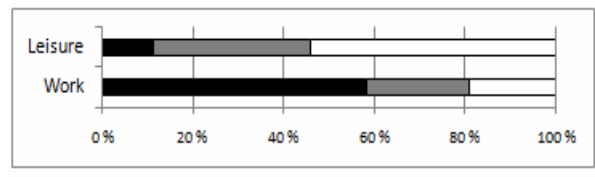

Employer

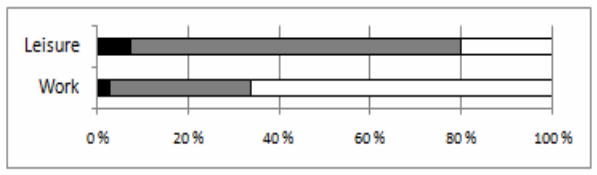

Merchant

Fig. 1. Distribution of faces based on inquirer (shown below) and situation (shown next to the bar). Black color denotes the True face, grey denotes the Vague face, and white the Blank face.

It is obvious that the "same face to same inquirer independently of situation" does not hold for our respondents. They were willing to reveal the True face to the employer only at work, not during leisure. Similarly, merchants would have mainly received a Blank face while the respondents were at work, but a Vague face during leisure. Targeted advertisements were considered potentially valuable if they were not distracting and if they did not require too detailed information about the receiver.

We then analyzed the effects of the demographic variables. The first variable we considered was gender. The distributions of the responses were similar by male and female respondents. There was only one case, shown in Figure 2 (left), where there was a difference by more than just a few percentages in the replies. Female employees were somewhat less willing to put up their true face to the employer. This was the case independently of whether the situation was at work or at leisure.
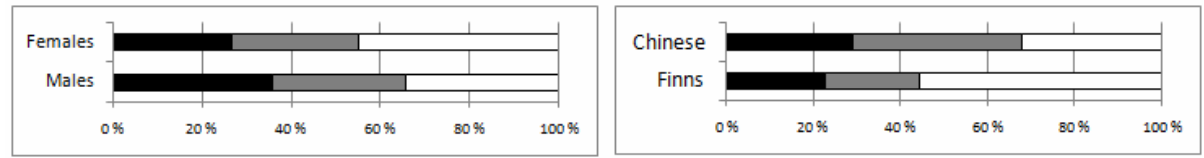

Fig. 2. Distribution of faces that female and male respondents would have shown to the employer (left), and Chinese and Finnish respondents would have shown at leisure (right)

Another interesting variable is the nationality of the respondents. The potential effect of nationality became clear only after reviewing the replies collected in 2004, and the nationality of respondents was systematically collected only in 2005; therefore the nationality-based analysis is based on a smaller sample than the ones above $(n=208)$.

The majority of respondents $(n=109)$ were Finns, so a natural first analysis was between Finns and non-Finns. The differences in the distributions were minor, but the non-Finns showed a lot of variation. The biggest subpopulation was formed by Chinese respondents $(n=25)$, and we compared the Finnish and Chinese respondents in more detail. Figure 2 (right) shows an example of typical results.

The biggest differences were that Chinese respondents would never show a Blank face to their spouse (as opposed to $6.4 \%$ of the Finns), and that the Chinese respondents would never show a True face to merchants (as opposed to $5.6 \%$ of all the other 
respondents). For strangers, $76.1 \%$ of the Finns would show a Blank face, whereas for Chinese the corresponding figure was $48.0 \%$.

\section{Discussion}

We requested our students to ask for explanations to the choices their respondents made. These revealed further variation and show clearly that the average and summary information given above must be taken only as indicative.

For instance, although Finns are considered as somewhat reserved (and indeed, did on average not have a problem with putting up a Blank face), there were exceptions. One Finnish respondent commented that "I've got nothing to hide," and another went even further: "I have to reveal, it's in my personality". When discussed in class, a student asked in disbelief: "Was that really a Finn?"

Another observation was that the faces chosen for the scenario were considered too general. People would have liked to know more about the inquirer. They would have been happy to put up at least a Vague face to merchants, had they known that the merchandise offered was potentially beneficial for them (e.g., an announcement of an interesting discount). They also might have put up a more revealing face than a Blank face to a stranger had they known something about the age and gender of the inquirer.

The results in Section 3 hint at the Chinese respondents being careful not to reveal too much of their identity when it was not necessary, but also not to put up a perhaps impolite Blank face. Finns, respectively, seemed less conscious and less considerate.

\section{Conclusions}

We repeated a study of privacy conceptions originally carried out in the USA. Our results show that one should be careful with general conclusions. A detailed analysis shows that the overall result of [2] does not hold in all cases. Culture was found to have a notable effect, and gender had an effect to a smaller degree. We are currently working on a more detailed analysis of the verbal comments of the participants.

Acknowledgments. We thank Scott Lederer for giving us access to the data set of the original study. This work was supported by Nordunet in the PriMa project (Privacy in the Making).

\section{References}

1. Lederer, S., Hong, J.I., Dey, A.K., Landay, J.A.: Personal Privacy through Understanding and Action: Five Pitfalls for Designers. Personal and Ubiquitous Computing 8(6), 440-454 (2004)

2. Lederer, S., Mankoff, J., Dey, A.K.: Who Wants to Know What When? Privacy Preference Determinants in Ubiquitous Computing. In: CHI 2003 Extended Abstracts on Human Factors in Computing Systems CHI 2003, pp. 724-725. ACM, New York (2003)

3. Lederer, S., Mankoff, J., Dey, A.K., Beckmann, C.: Managing Personal Information Disclosure in Ubiquitous Computing Environments. Technical Report CSD-03-1257. Univ. of California, Berkeley (2003), http: / / www . eecs . berkeley. edu / Pubs / TechRpts / $2003 / 5250$.html (retrieved January 28, 2009) 